\title{
ARTICLE \\ Polyclonal HIV envelope-specific breast milk antibodies limit founder SHIV acquisition and cell-associated virus loads in infant rhesus monkeys
}

\author{
Jonathon E. Himes ${ }^{1}$, Ria Goswami ${ }^{1}$, Riley J. Mangan ${ }^{1}$, Amit Kumar ${ }^{1}$, Thomas L. JeffriesJr. ${ }^{1}$, Joshua A. Eudailey ${ }^{1}$, Holly Heimsath ${ }^{1}$, \\ Quang N. Nguyen ${ }^{1}$, Justin Pollara ${ }^{1,2}$, Celia LaBranche ${ }^{1}$, Meng Chen ${ }^{1}$, Nathan A. Vandergrift ${ }^{1}$, James W. Peacock ${ }^{1}$, Faith Schiro ${ }^{3}$, \\ Cecily Midkiff ${ }^{3}$, Guido Ferrari ${ }^{1,2}$, David C. Montefiori ${ }^{1}$, Xavier Alvarez Hernandez ${ }^{3}$, Pyone Pyone Aye ${ }^{3}$ and Sallie R. Permar ${ }^{1,4,5,6}$
}

\begin{abstract}
Breast milk HIV-1 transmission is currently the predominant contributor to pediatric HIV infections. Yet, only 10\% of breastfeeding infants born to untreated HIV-infected mothers become infected. This study assessed the protective capacity of natural HIV envelope-specific antibodies isolated from the milk of HIV-infected women in an infant rhesus monkey (RM), tier 2 SHIV oral challenge model. To mimic placental and milk maternal antibody transfer, infant RMs were i.v. infused and orally treated at the time of challenge with a single weakly neutralizing milk monoclonal antibody (mAb), a tri-mAb cocktail with weakly neutralizing and ADCC functionalities, or an anti-influenza control mAb. Of these groups, the fewest tri-mAb-treated infants had SHIV detectable in plasma or tissues $(2 / 6,5 / 6$, and $7 / 8$ animals infected in tri-mAb, single-mAb, and control-mAb groups, respectively). Tri-mAb-treated infants demonstrated significantly fewer plasma transmitted/founder variants and reduced peripheral CD4+ T cell proviral loads at 8 weeks post-challenge compared to control mAb-treated infants. Abortive infection was observed as detectable CD4+ T cell provirus in non-viremic control $\mathrm{mAb}$ - and single $\mathrm{mAb}$-, but not in tri-mAb-treated animals. These results suggest that polyfunctional milk antibodies contribute to the natural inefficiency of HIV-1 transmission through breastfeeding and infant vaccinations eliciting non-neutralizing antibody responses could reduce postnatal HIV transmission.
\end{abstract}

Mucosal Immunology (2018) 11:1716-1726; https://doi.org/10.1038/s41385-018-0067-7

\section{INTRODUCTION}

According to the 2016 UNAIDS report, approximately 150,000 pediatric infections occur annually, accounting for $\sim 10 \%$ of new global human immunodeficiency virus (HIV)-1 infections. ${ }^{1}$ The benefits of breastfeeding to infant health are well recognized, yet vertical transmission of HIV-1 via breastfeeding results in nearly half of the annual mother-to-child-transmission (MTCT) occurrences. ${ }^{2}$ In resource-limited areas, formula-fed infants exhibit high mortality rates due to respiratory and diarrheal illnesses, ${ }^{3,4}$ and thus formula feeding is not a viable strategy to reduce pediatric HIV transmissions. While administration of antiretroviral therapy (ART) to HIV-1-infected, breastfeeding mothers reduces MTCT rates to $<5 \%,{ }^{5}$ socioeconomic barriers to ART access and compliance, ${ }^{6,7}$ as well as acute maternal infections, make it unlikely that ART alone can achieve eradication of pediatric HIV$1 .^{8-10}$ Therefore, developing effective immune-based prevention strategies, such as a maternal or infant vaccine to protect infants from oral HIV-1 acquisition during breastfeeding, may greatly contribute to the goal of achieving an HIV-free generation. ${ }^{11}$

Despite repeated, daily mucosal HIV exposure during years of breastfeeding, only $\sim 10 \%$ of breastfeeding infants of untreated HIV-infected mothers acquire HIV, ${ }^{11}$ suggesting the presence of protective factors in milk. The role of innate factors such as mucins, $^{12}$ defensins, ${ }^{13}$ lactoferrin, ${ }^{14}$ long chain fatty acids, ${ }^{15}$ interleukin-15, ${ }^{16}$ and tenascin $\mathrm{C}^{17}$ present in breast milk have been extensively studied for their anti-HIV activity. Additionally, the milk microbiome, particularly lactobacillus and pediococcal species, have been reported to inhibit HIV infection of target cells. ${ }^{18}$ In chronically HIV-infected mothers, breast milk also contains HIV-1 envelope (Env)-specific antibodies and Envspecific memory B cells, 19,20 which are primarily immunoglobulin G1 (IgG1) isotype and are otherwise similar in specificity and function to those identified in blood of chronically infected individuals. ${ }^{21}$ While breast milk antibodies capable of antibodydependent cellular cytotoxicity (ADCC) have been associated with diminished vertical transmission rates ${ }^{22}$ and reduced infant mortality after infection ${ }^{23}$ in humans, the protective capabilities of polyfunctional milk antibodies remain unclear. Induction or passive infusion of broadly neutralizing antibodies (bNAbs) is an attractive immunologic strategy for global HIV control (reviewed in ref. ${ }^{24}$ ) including in the setting of postnatal HIV transmission. $^{25,26}$ Yet, bNAbs only develop naturally in $<20 \%$ of individuals, typically take 2-4 years to develop after infection, ${ }^{27}$ and have been unable to be elicited through vaccination. Moreover, bNAbs

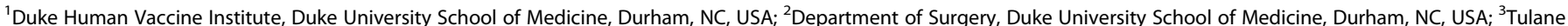

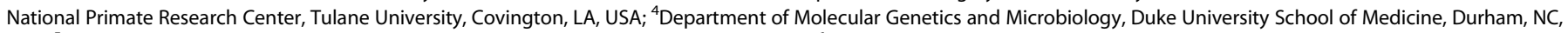

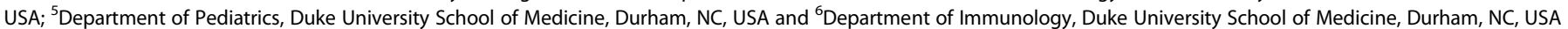
Correspondence: Sallie R. Permar (sallie.permar@duke.edu)

These authors contributed equally: Ria Goswami, Riley J. Mangan

Received: 15 January 2018 Revised: 18 June 2018 Accepted: 23 June 2018

Published online: 16 August 2018 
a

\begin{tabular}{|c|c|c|c|c|c|c|c|c|c|c|c|}
\hline \multirow{3}{*}{$\begin{array}{c}\text { Antibody } \\
\text { ID }\end{array}$} & \multirow{3}{*}{$\begin{array}{c}\text { Treatment } \\
\text { group }\end{array}$} & \multirow{3}{*}{ Env specificity } & \multirow{3}{*}{$\begin{array}{l}\text { Isotype and } \\
\text { class }\end{array}$} & \multirow{3}{*}{$\begin{array}{l}\text { ADCC endpoint } \\
\text { concentration } \\
(\mu \mathrm{g} / \mathrm{mL})\end{array}$} & \multicolumn{2}{|c|}{ Inhibition of: } & \multicolumn{5}{|c|}{ Neutralization IC $\mathrm{S}_{50}(\mu \mathrm{g} / \mathrm{mL})$ in TZM-bl cells: } \\
\hline & & & & & \multirow{2}{*}{$\begin{array}{c}\text { Dendritic cell } \\
\text { transfer }\end{array}$} & \multirow{2}{*}{$\begin{array}{c}\text { Epithelial cell } \\
\text { binding }\end{array}$} & \multicolumn{4}{|c|}{ Tier 1 viruses } & Tier 2 virus \\
\hline & & & & & & & SHIV SF162P4 & SHIV BaL-P4 & MW965.26 & SHIV-1157ipEL-p & SHIV-1157ipd3N4 \\
\hline \begin{tabular}{|l|l|} 
DH378 & \\
\end{tabular} & $m A B$ and tri-mAB & CD4 blocking & $\lg G 1$ & 0.5 & $100 \%$ & $96 \%$ & 1.07 & 6.09 & 0.04 & 13.37 & 79 \\
\hline DH382 & Tri-mAb & C1, A32-like & $\lg G 1$ & $<0.04$ & Undetectable & Undetectable & NT & NT & $>25$ & NT & NT \\
\hline DH377 & Tri-mAb & $\mathrm{V}_{3}$ & $\lg G 1$ & Undetectable & $100 \%$ & $98 \%$ & 24.21 & 0.42 & $<0.01$ & $<0.01$ & $>100$ \\
\hline
\end{tabular}

b

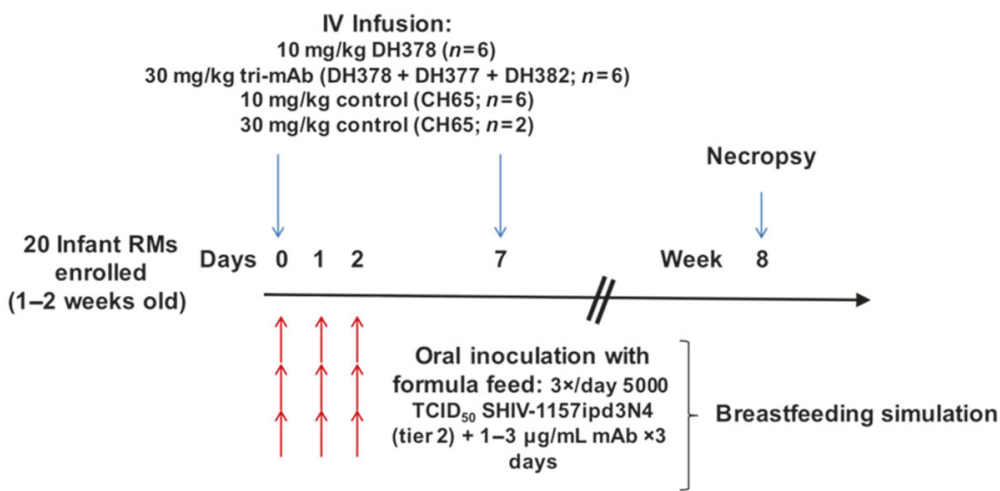

Fig. 1 Infant RM passive mAb infusion and oral SHIV challenge study design. a Functional characterization of maternal breast milk mAbs selected for passive infusion into infant RMs. MAbs were selected based on diverse functionality, including Env-binding specificity and ADCC, inhibition of dendritic cell virus transfer, inhibition of epithelial cell-virus binding, and virus neutralization functionalities. b Timeline for passive mAb infusion and SHIV-1157ipd3N4 challenge of infant RMs. Twenty infant RMs (1-2 weeks old) were systemically infused with the indicated mAbs and subsequently orally challenged after $1 \mathrm{~h}$ with $5000 \mathrm{TCID}_{50}$ of the tier 2 SHIV-1157ipd3N4 in formula feed to mimic infant oral viral exposure during breast feeding. Animals were reinfused 7 days after the first mAb infusion with the same mAb dose to sustain systemic Ab titers. Animals were necropsied at 8 weeks after challenge and lymphatic and GI tract tissues were collected

have not been identified in breast milk. ${ }^{19,28}$ Thus the contribution of non-neutralizing and weakly neutralizing breast milk antibodies to the inefficiency of HIV-1 transmission through breastfeeding warrants further exploration.

In this study, we sought to define the impact of systemic and orally administered natural breast milk-derived maternal HIV Envspecific monoclonal antibodies (mAbs) with non-neutralizing and weakly neutralizing functions on infant oral virus acquisition and dissemination in the periphery and lymphoid tissues. MAbs selected for this study were isolated from milk B cells of a cohort of HIV-1-infected Malawian women and were intended to represent IgG antibodies with various antiviral functionalities and specificities of those commonly found in breast milk (ADCC; tier 1 and weak tier 2 neutralization; dendritic cell-virus binding inhibition; epithelial cell-virus binding inhibition; and C1, V3, and CD4 blocking). ${ }^{19,28,29}$ Rhesus macaques (RMs) were passively infused with the maternal breast milk mAbs to mimic antibody transfer via the placenta and then repeatedly fed infant formula containing the maternal breast milk-derived mAbs and low-dose tier 2 chimeric simian/human immunodeficiency virus, SHIV1157ipd3N4. ${ }^{30}$ Defining the contributions of non-broadly neutralizing breast milk-derived antibodies to protection against transmission of HIV-1 through breastfeeding may inform the design of maternal and infant vaccines aimed at eliminating postnatal HIV-1 infections and limiting the size of the latent viral reservoir in the setting of breakthrough infections.

\section{RESULTS}

Selection of maternal breast milk mAbs for in vivo evaluation in infant monkeys and study design

The HIV-1 Env-specific mAbs isolated from breast milk B cells of lactating, HIV-1-infected Malawian women ${ }^{20}$ and selected for infusion into infant RMs in this study were initially characterized based on binding specificity, epithelial- and dendritic cell-virus binding inhibition, ADCC, and neutralization against the tier 2 challenge virus in this study, SHIV-1157ipd3N4, ${ }^{30}$ as well as neutralization of several tier 1 HIV/SHIV variants (Fig. 1a). ${ }^{28}$ As previously reported, all of the mAbs isolated from milk $B$ cells were
IgG1, did not demonstrate broadly neutralizing activity, and had similar characteristics to those isolated from peripheral blood HIV1 Env-specific B cells. ${ }^{20}$ The infusion mAbs were selected based on their diversity of functions and specificities; mAb DH378 demonstrated CD4-blocking capability associated with CD4-binding site (CD4bs) specificity and mAb DH377 bound to linear and conformational variable loop 3 (V3) binding, specificities previously associated with decreased MTCT risk, ${ }^{31,32}$ and mAb DH382 was constant region 1 (C1)-specific and competed with mAb A32Env binding. MAbs DH378 and DH377 were able to block epithelial- and dendritic cell-virus binding in vitro. ${ }^{28}$ Unsurprisingly, the A32-like mAb DH382 mediated potent ADCC activity against the tier 2 challenge SHIV-1157ipd3N4, with a maximum percentage of specific killing of $40.7 \%$ and an end point concentration of $<0.04 \mu \mathrm{g} / \mathrm{mL}$. Both DH378 and DH377 neutralized all tier 1 viruses tested, including SHIV SF162P4, SHIV BaL-P4, MW965.26, and SHIV-1157ipEL-p. Yet, DH378, but not DH377, demonstrated weak neutralization activity against the tier 2 clade C SHIV challenge virus when tested at high concentration (SHIV1157ipd3N4 neutralization; DH378 IC $\mathrm{I}_{50}=79 \mu \mathrm{g} / \mathrm{mL}$, DH377 IC $\mathrm{I}_{50}>$ $100 \mu \mathrm{g} / \mathrm{mL})$.

To assess the degree to which common HIV Env-specific polyfunctional milk antibodies contribute to protection against oral virus acquisition and replication, 20 infant RMs were divided into 2 anti-HIV-1 Env-specific breast milk mAb treatment groups $(n=6)$ and a control mAb treatment group $(n=8)$ for passive mAb infusion and oral SHIV-1157ipd3N4 challenge (Fig. 1b). In this study scheme, systemic passive infusion was intended to simulate placental lgG transfer, while the oral inoculum was intended to represent oral exposure to the combination of milk HIV Env-specific antibodies at physiologic levels with infectious virus. ${ }^{19}$ The first treatment group consisted of six animals treated with $\mathrm{DH} 378$, the weakly neutralizing $\mathrm{mAb}$ against the tier 2 challenge SHIV. The second group consisted of six animals treated with an equimolar mixture of mAbs $\mathrm{DH} 378$, $\mathrm{DH} 377$, and $\mathrm{DH} 382$ - a mixture containing mAbs with binding specificities including a specificity previously associated with decreased MTCT (V3), ${ }^{31,32}$ ADCC activity, dendritic- and epithelial cell-virus binding inhibition, and weak tier 2 neutralization against the challenge SHIV. Anti-influenza hemagglutinin (HA) mAb CH65 

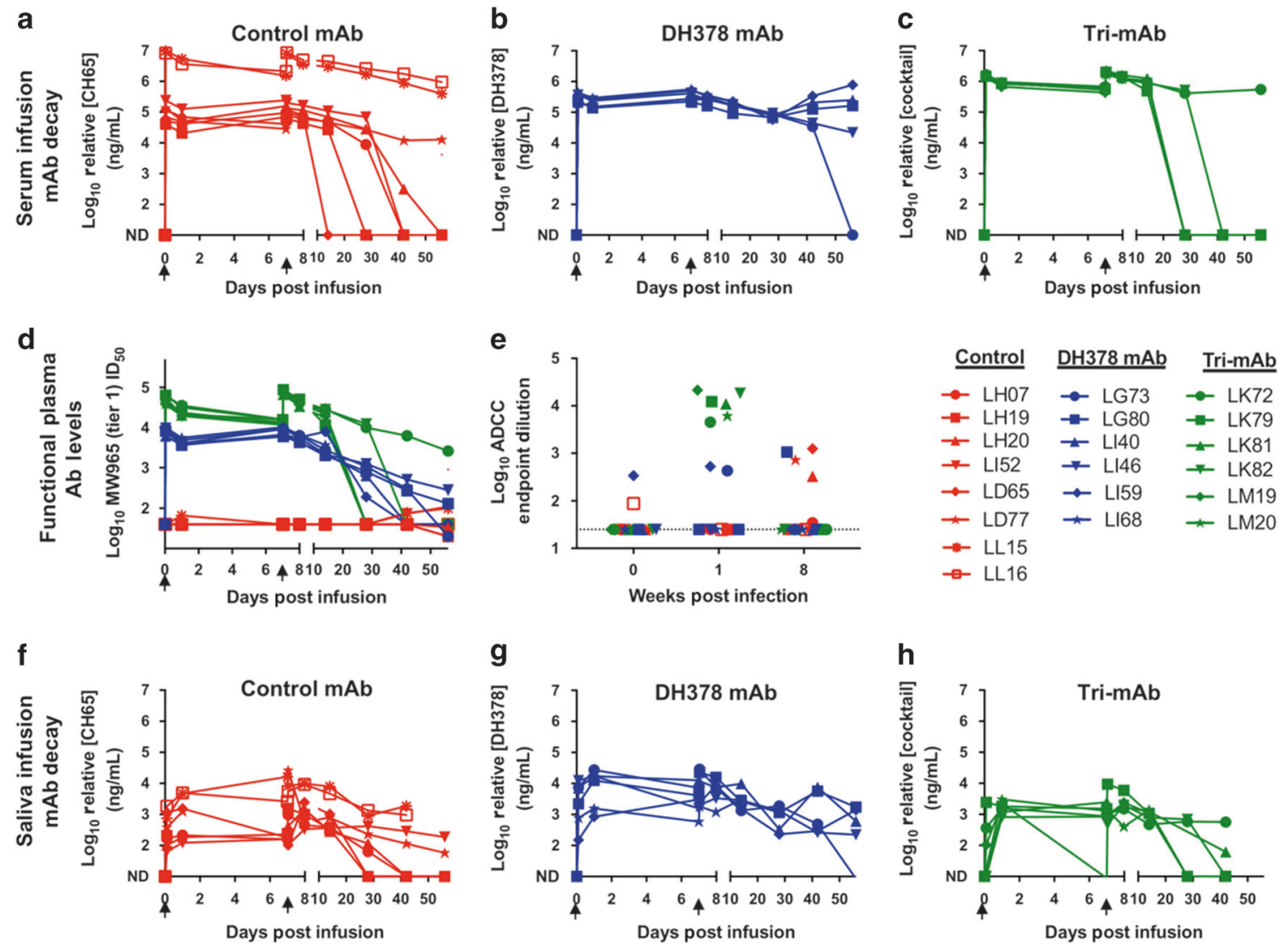

Fig. 2 The kinetics of passively infused mAbs and anti-SHIV Ab function in passively breast milk mAb-infused, SHIV-challenged infant RM serum and saliva. Concentrations of infused $\mathrm{mAbs}$ in $\mathbf{a}-\mathbf{c}$ serum and $\mathbf{f}-\mathbf{h}$ saliva from pre-infusion to 8 weeks post-infusion are depicted for control mAb-treated (a, f; CH65; red), DH378 mAb-treated (b, g; DH378; blue), and tri-mAb-treated (c, h; DH378, DH377, DH382; green) infant RMs. d Neutralizing Ab levels in serum reported as $\mathrm{ID}_{50}$ against the tier-1 virus MW965 from pre-infusion to 8 weeks post-challenge. e ADCCmediating $\mathrm{Ab}$ levels in serum reported as end point titer against SHIV-1157ipd3N4 at pre-infusion, 1 week (1 day after second mAb infusion), and 8 weeks post-challenge. Black arrows indicate systemic mAb infusions at days 0 and 7. ND not detectable

was employed as a control mAb treatment in 8 animals, including total $\mathrm{mAb}$ dose controls for each anti-HIV mAb treatment group (10 mg/kg CH65 in $n=6$ animals; $30 \mathrm{mg} / \mathrm{kg} \mathrm{CH65}$ in $n=2$ animals). To simulate placental maternal $A b$ transfer, infants were infused intravenously with the mAbs $1 \mathrm{~h}$ prior to the first of 9 oral SHIV1157ipd3N4/mAb combination challenges in formula (3 times/day for 3 consecutive days). Animals were reinfused with the same $\mathrm{mAb}$ dose on day 7 to sustain detectable systemic mAb levels for at least 2 weeks after virus challenge. SHIV-challenged infant monkeys were then followed for plasma mAb kinetics, systemic cell-free and cellassociated virus loads, and were necropsied at 8 weeks for assessment of lymphoid and gastrointestinal (Gl) tract tissue viral loads (VLs) (Fig. 1b).

Pharmacokinetics of maternal milk HIV-1 mAb infusion of infant RM serum and saliva

Serum mAb concentrations at longitudinal time points through 8 weeks post-SHIV-challenge were measured by enzyme-linked immunosorbent assay (ELISA) to determine the kinetics of the $m A b$ persistence. Serum mAb levels in animals from all treatment groups were similar through 2 weeks post-infusion, achieving peak concentrations of $9.2 \times 10^{4}-4.5 \times 10^{6} \mathrm{ng} / \mathrm{mL}$ and variable rates of $\mathrm{mAb}$ clearance, presumably due to variability of the infant immune response against the recombinant $\mathrm{mAb}$ and clearance rates (Fig. 2a-c; Figure S1).

Plasma NAb and ADCC levels were measured to assess in vivo functionality of the infused treatment mAbs and the development of host humoral responses (Fig. 2b, c). Predictably, NAb responses against the tier 1 clade $\mathrm{C}$ virus MW965 were high in all DH378- and
tri-mAb-treated animals early after $m A b$ infusion, and the magnitude of the responses declined to undetectable in some animals as early as 6 weeks post-challenge when circulating $\mathrm{mAb}$ levels declined (Fig. 2d). Interestingly, several control animals (LH20, LI52, LD65, LD77, LL15) developed tier 1, MW965 NAb responses as early as 6 weeks post-challenge. None of the animals had detectable plasma neutralization against either the tier 2 SHIV-1157ipd3N4 challenge virus or the related tier 1 virus SHIV1157ipEL- $\mathrm{p}^{33}$ at peak mAb concentrations or 8 weeks postchallenge, which was unsurprising as serum mAb concentrations never surpassed that of the in vitro $\mathrm{IC}_{50}$ (Fig. 1a). Serum ADCC end point titers against SHIV-1157ipd3N4-infected CD4+ T cells were measured pre-infusion, at peak infusion mAb concentration (1 day after second infusion), and at 8 weeks postinfection for control and mAb-treated RMs (Fig. 2e). As expected, serum from tri-mAbtreated animals exhibited high-magnitude ADCC function (mean end point dilution $=12,303$ ), which deteriorated by 8 weeks postinitial infusion to undetectable levels. Two of the six DH378treated animals demonstrated low-magnitude ADCC function detectable at peak mAb levels (mean end point dilution $=175$ ), comparable to that of background levels detected in some infants (Fig. 2e). Notably, two of the eight control mAb-treated and one of the six DH378-treated animals, but none of the tri-mAb-infused infant RMs, developed detectable autologous ADCC-mediating $\mathrm{mAbs}$ against the challenge SHIV strain after infection at 8 weeks post-initial infusion, presumably related to virus load at 8 weeks of infection.

Saliva mAb concentrations were also measured prior to and following oral challenge (Fig. $2 f-h$ ). All animals demonstrated 
a

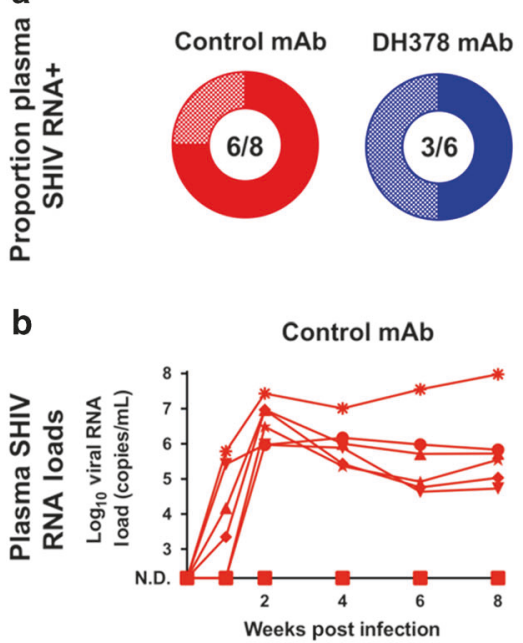

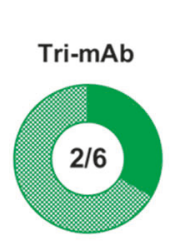

DH378 mAb $\underline{\text { Tri-mAb }}$

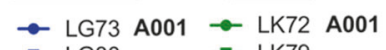

$\rightarrow$ LG80 $\rightarrow$ LK79

\# L140 \# LK81 A001

$\rightarrow$ LI46 $\rightarrow$ LK82 A001

$\rightarrow$ LI59 A001 $\rightarrow$ LM19

- Ll68 $\rightarrow$ LM2O

* LD77

$\rightarrow$ - LL15

$\rightarrow$ LL16

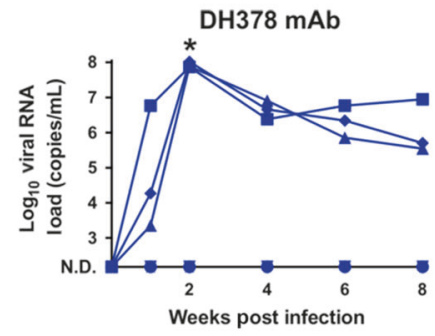

Tri-mAb

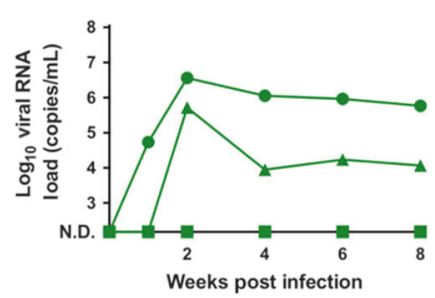

Fig. 3 Infection rates and plasma viral loads of passively breast milk mAb-infused, SHIV-challenged infant RMs. a Proportion of viremic RMs with 6/8 control mAb-treated RMs (red), 3/6 DH378 mAb-treated RMs (blue; Fisher's exact test; FDR-corrected $p=0.58$ ), and 2/6 tri-mAbtreated RMs (green; Fisher's exact test; FDR-corrected $p=0.55$ ) demonstrating viremia as defined by a detectable plasma viral RNA load at any tested time point. b Plasma viral RNA loads in mAb treated, orally SHIV challenged infant RMs. Asterisk $(*)$ indicates significantly higher 2 week postinfection plasma viral RNA loads in DH378 mAb-treated RMs compared to CH65-treated RMs (Wilcoxon test; FDR-corrected $p=0.05$ ). Animals with HLA-type A001 or B17 are annotated appropriately in the legend

detectable mAb levels in saliva at $1 \mathrm{~h}$ post-infusion, the time of the first SHIV challenge. Saliva mAb levels peaked around 1 day postinfusion in all animals and only slightly declined by 7 days postinitial infusion in most animals.

Effect of maternal milk HIV-1 antibody treatment on SHIV acquisition and plasma viremia in orally challenged infant RMs To characterize the effectiveness of the mAb treatments in preventing SHIV acquisition, SHIV plasma viral RNA loads of orally challenged infant RMs were measured in longitudinal samples by quantitative reverse transcriptase (qRT-PCR). In all, 6/8 (75\%) CH65-treated control animals, 3/6 (50\%) DH378-treated animals, and 2/6 (33\%) tri-mAb-treated animals became detectably viremic after 9 oral SHIV challenges, resulting in statistically similar systemic SHIV acquisition rates between controls and each treatment group (Fig. 3a; false discovery rate (FDR)-corrected Fisher's exact test; controls vs. DH378 $p=0.58$, tri-mAb cocktail $p$ $=0.55$ ). Peak and set point viral RNA loads in plasma were largely similar between $\mathrm{CH} 65$-treated control animals (median and range; peak $=6.2 \times 10^{6}$ copies $/ \mathrm{mL}, \quad 9.3 \times 10^{5}-2.7 \times 10^{7}$ copies $/ \mathrm{mL} ; \quad$ set point $=4.4 \times 10^{5}$ copies $/ \mathrm{mL}, 5.4 \times 10^{4}-9.5 \times 10^{7}$ copies $/ \mathrm{mL}$ ) and tri$\mathrm{mAb}$-treated animals (median and range; peak $=2.1 \times 10^{6}$ copies/ $\mathrm{mL}, 5.3 \times 10^{5}-3.6 \times 10^{6}$ copies $/ \mathrm{mL}$; set point $=3.0 \times 10^{5}$ copies $/ \mathrm{mL}$, $1.2 \times 10^{4}-5.9 \times 10^{5}$ copies $/ \mathrm{mL}$ ) when excluding animals with undetectable plasma viral RNA loads (Fig. 3b). Interestingly, the tri-mAb cocktail-treated animal LK81 emerged as an outlier with the lowest plasma peak and set point viral RNA loads observed in a viremic animal from this study (peak $=5.3 \times 10^{5}$ copies $/ \mathrm{mL}$; set point $=1.2 \times 10^{4}$ copies $/ \mathrm{mL}$ ). While set point viral RNA loads in plasma were similar between viremic $\mathrm{CH} 65$-treated control animals and viremic DH378-treated animals (median and range; peak $=7.7 \times 10^{7}$ copies $/ \mathrm{mL}, \quad 7.4 \times 10^{7}-1.1 \times 10^{8}$ copies $/ \mathrm{mL}$; set point $=5.1 \times 10^{5}$ copies $/ \mathrm{mL}, \quad 3.5 \times 10^{5}-8.9 \times 10^{6}$ copies $/ \mathrm{mL}$ ), the peak viral RNA loads in plasma from viremic DH378-treated animals was significantly higher than that from viremic controls (Fig. 3b; FDR-corrected Wilcoxon test; FDR-corrected $p=0.05$ ). Given the ranges of peak and set point viral RNA loads, major histocompatibility complex (MHC) typing was performed to rule out unbalanced MHC types associated with natural control of viremia. Several infants possessed MHC alleles highly (MamuA001, Mamu-B017) or weakly (Mamu-A002, Mamu-B047) associated with low set point VLs and/or longer survival lengths (Table 1). ${ }^{34-41}$ Yet, these protective alleles were not more common in any single treatment group (67\% for control mAb- and tri-mAbtreated animals and $80 \%$ for $\mathrm{DH} 378$ mAb-treated animals had least one protective MHC allele). Additionally, all groups also contained animals with the Mamu-A004 allele, which is associated with increased set point VLs. ${ }^{42}$ All viremic, DH378 mAb-treated animals possessed this allele, which could contribute to the significantly higher plasma VLs observed in these animals (Fig. 3b).

Cell-associated SHIV load in blood and tissues from breast milk mAb-treated, orally SHIV-challenged infant RMs

To determine whether the presence of pre-existing HIV-1 Envspecific mAbs impacted the cell-associated VL in blood and tissue compartments following oral challenge in mAb-infused infant RMs, tissue mononuclear cell proviral loads, proportions of viral RNA producing T cells, and tissue-associated infectious virus titers were measured in peripheral blood mononuclear cells (PBMCs), GI tract, and lymphoid tissues (Fig. 4). SHIV provirus in CD4+ T cells in PBMCs (preinfection, 2 and 8 weeks postinfection), Gl, and lymphoid tissues at 8 weeks post-challenge in viremic infants demonstrated widely variable but routinely detectable proviral loads $\left(10^{4}-10^{7}\right.$ gag copies/million CD4+ T cells) independent of tissue type or mAb treatment (Fig. 4a). SHIV provirus was undetectable in PBMCs or any tissues isolated from tri-mAbtreated animal LK81, which exhibited detectable, albeit relatively low plasma SHIV RNA loads.

The proportion of SHIV RNA-transcribing cells as measured by in situ hybridization assays was also similar in lymphoid and $\mathrm{Gl}$ tissues from animals in all treatment groups. However, mesenteric lymph node (LN) and spleen from control mAb-treated animal LL15 exhibited a notably higher prevalence of SHIV RNA-positive cells compared to other SHIV-infected infants (Fig. 4b). Extensive SHIV RNA production was observed within and surrounding Peyer's patches of the small intestine as well as in CD3 + T cells of lymphoid tissues (Fig. 4c). Additionally, SHIV RNA detection within the Peyer's patches largely failed to colocalize with CD3 expression, indicating viral association with dendritic cells and/or other antigen-presenting cells within these germinal centers.

To assess the level of cell-associated infectious SHIV within oralassociated lymphoid and GI tissues at 8 weeks post-challenge, 
Table 1. MHC alleles present in control mAb-, DH378 mAb-, and tri-mAb-treated and SHIV-1157ipd3N4-challenged infant RMs

\begin{tabular}{|c|c|c|c|c|c|c|c|c|}
\hline \multirow[t]{2}{*}{ Animal ID } & \multirow[t]{2}{*}{ Treatment group } & \multirow[t]{2}{*}{ Infection status } & \multicolumn{2}{|l|}{ Mamu-A } & \multicolumn{2}{|l|}{ Mamu-B } & \multicolumn{2}{|l|}{ Mamu-DRB } \\
\hline & & & Haplotype 1 & Haplotype 2 & Haplotype 1 & Haplotype 2 & Haplotype 1 & Haplotype 2 \\
\hline LH07 & Control $\mathrm{mAb}$ & Viremic & $A 001$ & A008 & B055 & B024a & DR01a & DR14a \\
\hline LH20 & & & $A 002 a$ & A004 & B001a & B001a & DR01a & DR016 \\
\hline LI52 & & & A006 & $\mathrm{A} 224 \mathrm{a}$ & В015a & $B 017 a$ & DR03f & DR016 \\
\hline LL15 & & & A007 & A008 & B001a & B055 & DR04a & DR06 \\
\hline LH19 & & Non-viremic & A004 & A008 & B021a & B047a & DR01a & DR06 \\
\hline LL16 & & & A004 & A008 & B012b & B080 & DR03a & DR11a \\
\hline LG80 & $\mathrm{DH} 378 \mathrm{mAb}$ & Viremic & A004 & $\mathrm{A} 011$ & B055 & B012b & DR11c & DR02' \\
\hline LI59 & & & $A 001$ & $\mathrm{~A} 004$ & В024a & B048 & DR03a & DR03f \\
\hline LI40 & & & $A 002 a$ & A004 & B012a & B012a & DR03a & DR03f \\
\hline LG73 & & Non-viremic & $A 001$ & A011 & B055 & B001a & DR01a & DR04a \\
\hline LI46 & & & A002a & A002a & B048 & B048 & DR04a & DR03f \\
\hline LK72 & Tri-mAb & Viremic & $A 001$ & $A 002 a$ & B024a & B012a & DR03f & DR03f \\
\hline LK81 & & & $A 001$ & A008 & B043a & B047a & DR01a & DR13a \\
\hline LK79 & & Non-viremic & $A 002 a$ & $\underline{\mathrm{A} 004}$ & B001a & B012a & DR11a & DR03f \\
\hline LK82 & & & A002a & $\overline{A 001}$ & B001a & B001a & DR11a & DR01a \\
\hline LM19 & & & $\underline{\mathrm{A} 004}$ & $\underline{\mathrm{A} 004}$ & B001a & B002 & DR03a & DR13a \\
\hline LM20 & & & A004 & A008 & В015a & B015b & DR03a & DR01a \\
\hline
\end{tabular}

Characters in italics and bold indicate MHC alleles previously reported as highly ${ }^{33-40}$ or mildly ${ }^{33,35,38,39}$ associated with lower plasma set point viral load, respectively. Underlining indicates MHC alleles associated with higher plasma viral load at set point ${ }^{41}$

tissue mononuclear cells were cocultured with TZM-bl reporter cells with luminescence output indicating the level of infectious SHIV production (Figure S2). In general, tissue-associated infectious SHIV titers were similar between treatment groups (Fig. 4d). However, the palatine tonsil exhibited the highest cell-associated infectious titers of any tissue in four of the seven infants with sufficient tonsil tissue for the assay. Furthermore, this assay employed total mononuclear cells and flow cytometric analysis revealed that proportions of $\mathrm{CD} 4+\mathrm{T}$ cells of total $\mathrm{CD} 45+$ mononuclear cells were lower in palatine tonsil than other lymphoid tissues (Figure S3), indicating a potential highly infectious tissue-associated virus titer in palatine tonsil. Additionally, two viremic animals, CH65-treated animal LHO7 and tri-mAbtreated animal LK81, exhibited undetectable tissue-associated infectious virus titers.

Proviral loads, tissue-associated infectious virus titers, and tissue viral RNA levels in PBMCs (preinfection, 2 and 8 weeks postinfection), Gl tract tissues, and lymphoid tissues in nonviremic, SHIV-challenged infant RMs were also measured to assess the possibilities of abortive infection or low-level replication in the non-viremic infant RMs (Fig. 5). Several animals with undetectable plasma SHIV RNA loads throughout the study including DH378-treated animals LG73 and LI46, and CH65-treated animal LH19, had detectable SHIV proviral DNA loads in various tissues at similar levels to those measured from animals with detectable plasma SHIV RNA loads (Fig. 5a). To confirm the presence of tissue-associated virus in these nonviremic animals, the SHIV proviral envelopes were amplified by PCR of genomic DNA (gDNA) from submandibular LN CD4+ T cells of LH19 and LG73, sequenced to demonstrate the presence of full-length envelope open reading frames, and expressed as pseudovirus in the SG3Denv backbone to assess env infectious functionality (Fig. 5b). Both pseudoviruses were capable of infecting TZM-bl reporter cells with the LH19 and LG73 env pseudoviruses eliciting luminescence magnitudes of 571,294 relative luminescence units (RLU) and 176,820 RLU, respectively, which was appreciably higher than that of cell only controls ( $\sim 500 \mathrm{RLU})$.
Thus four infants, at least one from each $\mathrm{mAb}$ treatment group, had discordance between the presence of detectable plasma viral RNA and CD4+ T cell provirus (Fig. 6a). Provirus was observed in CD4+ $T$ cells isolated from non-viremic animals LG73, LI46, and LH19. Alternatively, provirus was not detectable in the viremic animal LK81. Notably, prior to correction for multiple comparisons, tri-mAb-treated infants, but not DH378-treated infants, demonstrated lower magnitude proviral loads in 8-week PBMCs compared to control mAb-treated animals (Wilcoxon test; raw $p$ $=0.03$; FDR-corrected $p=0.18$ ). Taken together, 7/8 CH65-treated control animals, 5/6 DH378-treated animals, and 2/6 tri-mAbtreated animals demonstrated either detectable viremia or proviral loads following oral SHIV challenge (Fig. 6b).

Plasma SHIV variant diversity in mAb-treated and orally SHIVinfected infant RMs

To assess the potential impact of maternal mAb passive infusion of infants on the genetic bottleneck of mucosal virus transmission and SHIV env diversification by peak viremia ( 2 weeks postchallenge), single SHIV variants were isolated from infant RM plasma using single genome amplification (SGA). Of note, the median viral diversity of the SHIV-1157ipd3N4 challenge virus stock was $0.02 \%$ (range $=0-0.07 \%$ ), considerably lower than the diversity typically observed in chronically HIV-1-infected humans ( $>1 \%$ diversity). Viral diversity within each animal was visualized with phylogenetic tree and highlighter plot (Figure S4). Distinct SHIV transmitted/founder (T/F) variants present in plasma isolated at peak viremia ( 2 weeks post-challenge) were enumerated using previously established criteria, ${ }^{43}$ requiring that a genotypically distinct variant contain two novel mutations (to minimize the possibility of recombination yielding false results), and that these mutations be observed in at least 2 SGAs (to minimize the effects of PCR-related mutation). A similar number of distinct SHIV variants were present in the plasma at peak viremia between control mAb-treated RMs (Table $2 ; n=8$; median variants isolated $=2.5$; range $=0-4)$ and $\mathrm{DH} 378$ mAb-treated animals $(n=6$; median variants isolated $=0.5$; range $=0-2$; Wilcoxon test; raw $p=0.16$ ). However, plasma SHIV diversity at peak viremia was 

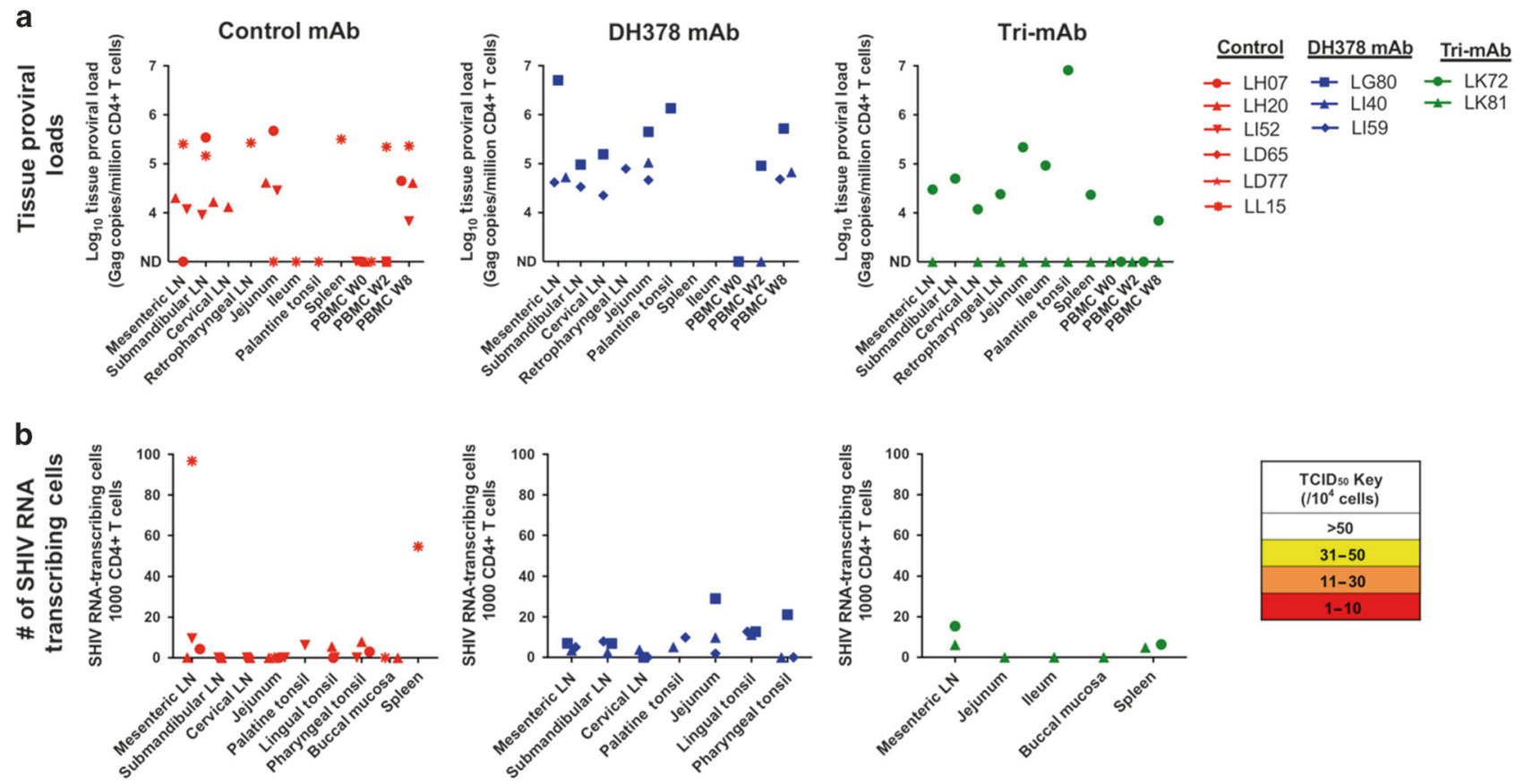

C

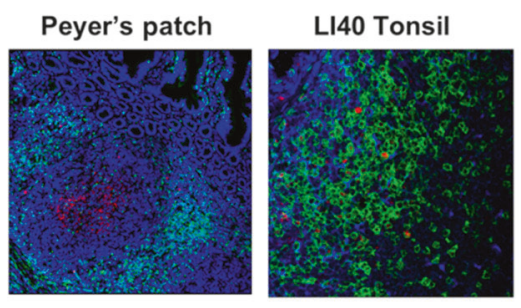

\begin{tabular}{|c|c|c|c|c|c|c|c|c|}
\hline \multirow{2}{*}{ Group } & \multirow{2}{*}{ PTID } & \multicolumn{7}{|c|}{ Tissue-associated infectious SHIV titer $\left(110^{\wedge} 4\right.$ cells) } \\
\hline & & Mes LN & Cer LN & Retro LN & Sub LN & Pal Ton & Jejunum & Spleen \\
\hline \multirow{4}{*}{$\begin{array}{c}\text { Control } \\
\mathrm{mAb}\end{array}$} & LH07 & 100 & $>63$ & NT & $>50$ & $>100$ & $>163$ & NT \\
\hline & LH20 & 33.3 & $>100$ & NT & 25.0 & 10.0 & $>160$ & NT \\
\hline & LI52 & $>400$ & NT & 200 & $>400$ & 100 & $>400$ & $>400$ \\
\hline & LL15 & 8.9 & $>95$ & NT & 7.4 & $>24.1$ & $>425$ & 20.0 \\
\hline \multirow{3}{*}{$\begin{array}{c}\text { DH378 } \\
\text { mAb }\end{array}$} & LG 80 & 41.2 & 58 & NT & 15.0 & 8.8 & 170 & NT \\
\hline & LI40 & 36.4 & $>100$ & NT & 17.4 & 9.1 & $>200$ & 57 \\
\hline & LI59 & 100 & 80 & 36.4 & 46.7 & NT & 70 & 40.0 \\
\hline \multirow{2}{*}{ Tri-mAb } & LK72 & 17.4 & 150 & 25.0 & 22.2 & $>22$ & 5.9 & $>400$ \\
\hline & LK81 & $>400$ & $>120$ & $>400$ & $>200$ & $>250$ & $>400$ & $>400$ \\
\hline
\end{tabular}

Fig. 4 Tissue-associated SHIV measures in mononuclear cells isolated from lymphoid and Gl tissues of viremic infant RMs 8 weeks postpassive breast milk mAb infusion and oral SHIV challenge. a CD4+ T cell proviral DNA loads reported as copy number/million CD4+ T cells in lymphoid and GI tissue mononuclear cells and $\mathbf{b}$ the number of SHIV RNA-producing CD3+ T cells in lymphoid and Gl tissue blocks reported as the number of SHIV Gag RNA+ cells per 1000 CD3+ T cells were measured for control mAb-treated (red), DH378 mAb-treated (blue), and tri-mAb-treated RMs (green) through ddPCR and in situ hybridization, respectively. ND not detectable. c Representative images of stained tissue blocks demonstrating active SHIV RNA transcription within a Peyer's Patch (LI40 jejunum) and in CD3+ T cells in lymphoid tissue (LI40 lingual tonsil) employed to assess the number of SHIV RNA-transcribing cells within lymphoid and GI tissues. Blue, green, and red depict nuclear, CD3, and SHIV Gag RNA staining, respectively. d Tissue-associated infectious SHIV-1157ipd3N4 titers measured through tissue mononuclear cell coculture with TZM-bl reporter cells. Reported titers represent the estimated minimum number of mononuclear cells required to yield detectable infection (cutoff defined by mononuclear cells from unchallenged RM-negative controls) of the TZM-bl cells in $50 \%$ of replicates. The cutoff for the limit of detection varied for each sample and was determined by mononuclear cell availability. NT indicates untested samples

lower in tri-mAb-treated RMs $(n=6$; median variants isolated $=0$; range $=0-1$; Wilcoxon test; raw $p=0.05$ ), indicating a possible sieve effect of the tri-mAb treatment on oral SHIV acquisition. SHIV env amplicons from all infants in a single tree rooted to the SHIV1157ipd3N4 challenge virus env revealed that the tri-mAb-treated RMs LK72 and LK81 are more homogenous compared to the other two infusion groups (Figure S5).

\section{DISCUSSION}

Despite daily chronic oral exposure to HIV for up to 2 years, only $\sim 10 \%$ of breastfeeding infants of untreated HIV-infected mothers acquire HIV. ${ }^{11}$ Furthermore, postnatally HIV-infected infants demonstrate slower disease progression compared to in utero or peripartum infected infants. ${ }^{44}$ Therefore, a better understanding of the naturally protective components in breast milk of HIVinfected mothers, including milk antibodies, could aid in the development of effective vaccination strategies aimed at reducing MTCT and other modes of HIV acquisition. HIV-specific NAbs have been shown to reduce chronic VLs and enhance host anti-HIV B cell responses in orally challenged, infected RM infants. ${ }^{45}$ Additionally, post-exposure infusion of an anti-HIV bNAb cocktail within $24 \mathrm{~h}$ after infection was capable of SHIV clearance in infant RMs. $^{26}$ While tier 1 virus weakly NAbs predicted reduced MTCT rate, $^{32}$ maternal plasma HIV-specific antibody potency and breadth has recently been associated with increased incidence of vertical transmission. ${ }^{46}$ Yet, HIV-specific ADCC-mediating antibody levels have been associated with reduced vertical transmission rates and infant mortality. ${ }^{22,23}$ Thus the potential protective effects of maternal breast milk antibodies lacking neutralization breadth and potency remains uncertain. In this study, we sought to probe the protective capabilities of several weakly or nonneutralizing, polyfunctional milk mAbs previously isolated from a cohort of HIV-infected Malawian women in an infant RM passive infusion and oral SHIV challenge model. The three maternal mAbs selected for inclusion in this study were intended to represent mAbs exhibiting various anti-HIV functionalities (ADCC, tier 1 and weak tier 2 neutralization, dendritic cell-virus binding inhibition, epithelial cell-virus binding inhibition) and HIV Env specificities (C1, V3, CD4 blocking). The treatment groups in this study were 

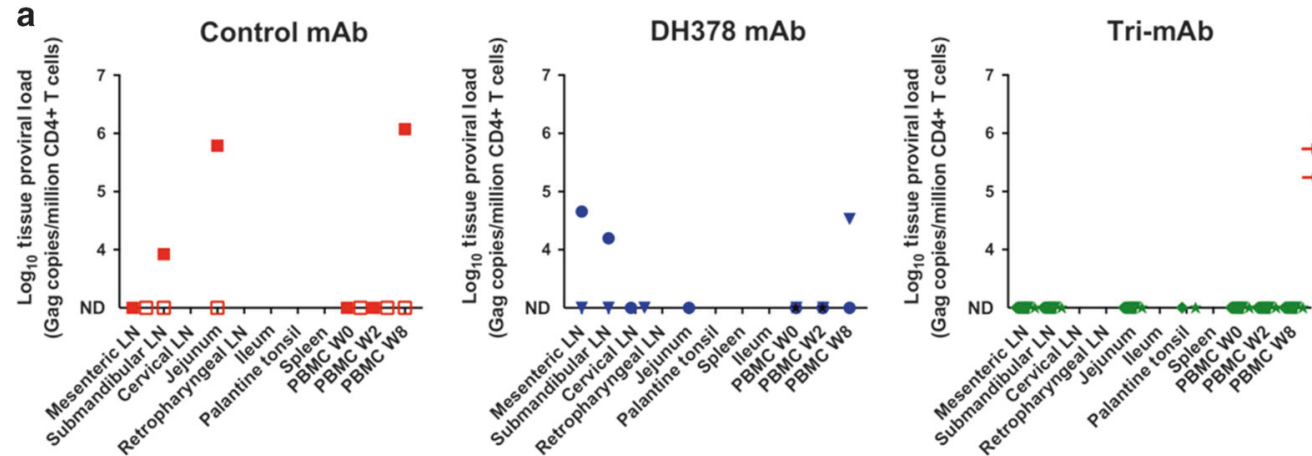

$$
\begin{aligned}
& \text { Control DH378 mAb Tri-mAb } \\
& \rightarrow \text { LH19 } \rightarrow \text { LG73 } \rightarrow \text { LK79 } \\
& \rightarrow \text { LL16 } \rightarrow \text { LI46 } \rightarrow \text { LK82 } \\
& \rightarrow \mathrm{L} 168 \rightarrow \mathrm{LM} 19 \\
& \text { - LM20 }
\end{aligned}
$$

b

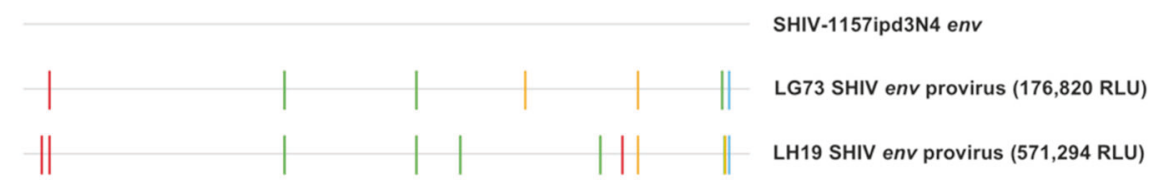

$0 \quad 200 \quad 400 \quad 600 \quad 800 \quad 1000 \quad 1200 \quad 1400 \quad 1600 \quad 1800 \quad 2000 \quad 2200 \quad 2400 \quad 2600$

Alignment position

Fig. 5 Tissue-associated SHIV DNA in CD4+ T cells isolated from lymphoid and Gl tissues of non-viremic infant RMs 8 weeks post-passive breast milk mAb infusion and oral SHIV challenge. a Proviral DNA loads reported as copy number/million CD4+ T cells in lymphoid and Gl tissue mononuclear cells were measured for control mAb-treated (red), DH378 mAb-treated (blue), and tri-mAb-treated RMs (green) through ddPCR. ND not detectable. b Highlighter plot alignments of two amplified SHIV env sequences obtained through SGA of extracted DNA from CD4+ T cells isolated from LH19 and LG73 submandibular lymph nodes compared to the SHIV-1157ipd3N4 env stock sequence. Colored lines indicate nucleotide mismatches compared to the SHIV stock. These envs were cloned and subsequently incorporated into pseudoviruses with a SG3Aenv backbone through co-transfection in 293T cells. Pseudovirus infectivity was estimated in TZM-bl reporter cells as RLUs compared to

\begin{tabular}{|c|c|c|c|c|c|c|c|c|}
\hline $\begin{array}{l}\text { Control mAb } \\
\text { (animal ID) }\end{array}$ & $\begin{array}{l}\text { No. of } \\
\text { variants }\end{array}$ & $\begin{array}{l}\text { SGA sequences } \\
\text { per animal }\end{array}$ & $\begin{array}{l}\mathrm{DH} 378 \mathrm{mAb} \\
\text { (animal ID) }\end{array}$ & $\begin{array}{l}\text { No. of } \\
\text { variants }\end{array}$ & $\begin{array}{l}\text { SGA sequences } \\
\text { per animal }\end{array}$ & $\begin{array}{l}\text { Tri-mAb } \\
\text { (animal ID) }\end{array}$ & $\begin{array}{l}\text { No. of } \\
\text { variants }\end{array}$ & $\begin{array}{l}\text { SGA sequences } \\
\text { per animal }\end{array}$ \\
\hline LI52 & 4 & 33 & LG80 & 2 & 30 & LK72 & 1 & 25 \\
\hline LH2O & 3 & 23 & LI40 & 1 & 21 & LK79 & 0 & - \\
\hline LL15 & 3 & 27 & LI46 & 0 & - & LK82 & 0 & - \\
\hline LH07 & 2 & 16 & LG73 & 0 & - & LM19 & 0 & - \\
\hline LH19 & 0 & - & & & & & & \\
\hline LL16 & 0 & - & & & & & & \\
\hline $\begin{array}{l}\text { Median (range) } \\
\text { no. of variants }\end{array}$ & $2.5(0-4)$ & & $\begin{array}{l}\text { Median (range) } \\
\text { no. of variants }\end{array}$ & $0.5(0-2)$ & & $\begin{array}{l}\text { Median (range) } \\
\text { no. of variants }\end{array}$ & $0(0-1)$ & \\
\hline
\end{tabular}
cell-only controls ( $<500 \mathrm{RLU})$. Pseudoviruses expressing env isolated from LH19 and LG73 demonstrated infectious capability with 571,294 RLU and 176,820 RLU magnitudes, respectively

Table 2. Diversity of SHIV-1157ipd3N4 variants in serum isolated 1 week post-initial oral challenge from control mAb-, DH378 mAb-, or tri-mAbtreated infant RMs

designed to assess whether a CD4-blocking mAb with tier 1 and weak autologous tier 2 neutralization ( $\mathrm{DH} 378$ mAb treatment group) or a polyclonal $\mathrm{mAb}$ infusion with multiple anti-viral functionalities (tri-mAb treatment group) could contribute to protection.

As expected, serum from groups treated with mAbs DH377 and/ or DH378 exhibited neutralization against tier 1 virus, MW965, and serum from groups treated with mAb DH382 exhibited ADCC against the challenge SHIV. The lack of SHIV-1157ipd3N4 neutralization by $\mathrm{DH} 378$-infused animals was expected as $\mathrm{mAb}$ levels of $\mathrm{DH} 378$ did not reach the $\mathrm{IC}_{50}$ for SHIV-1157ipd3N4 neutralization $(79 \mu \mathrm{g} / \mathrm{mL})$, given the limitation of the infusion volume in infant RMs. Of note, this in vitro estimation of neutralization potency in TZM-bl reporter cells does not necessarily directly predict the in vivo effect of infused mAbs. ${ }^{47}$ Several viremic animals treated with the control mAb developed neutralization responses against MW965 and ADCC responses against the challenge SHIV by week 8 . Yet, only one viremic animal from both anti-HIV mAb-treated groups exhibited adaptive ADCC responses at week 8 , which could indicate mAb blocking of immunogenic epitopes.

Plasma viremia was assessed to classify the extent to which anti-HIV breast milk mAb-treated animals were protected from systemic SHIV acquisition relative to the control mAb $\mathrm{CH} 65$ infused animals. Importantly, as maternal mAbs employed in this study did not coevolve with the challenge virus, which would be the case in the natural setting of infant HIV-1 exposure, the observed protection facilitated by these mAbs may underrepresent that which would occur against autologous viral strains. Both the $\mathrm{DH} 378$ treatment and the tri-mAb cocktail treatment groups had a lower number of viremic animals compared to the control mAb-infused group (6/8 animals viremic), with only $3 / 6$ 

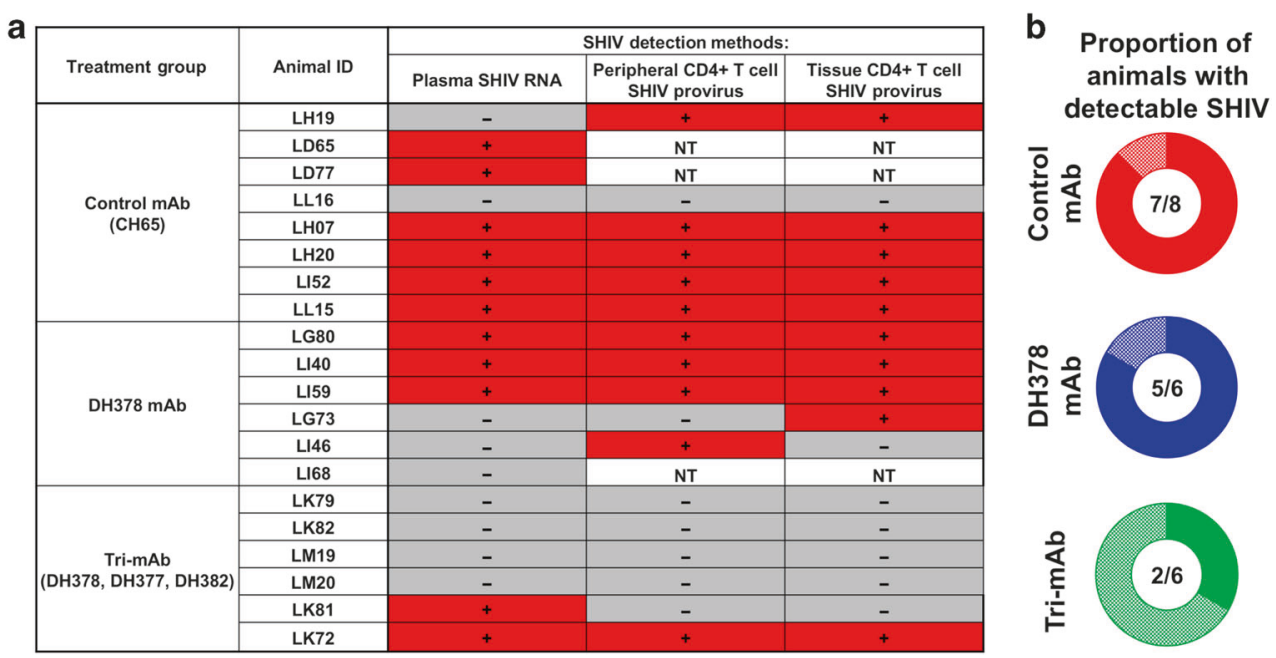

Fig. 6 Summary of SHIV detection and proportion of animals with detectable cell-free or cell-associated SHIV in breast milk mAb-infused, orally SHIV-challenged infant RMs. a Binary indication of detectable plasma SHIV RNA, peripheral CD4+ T cell SHIV provirus, and tissue CD4+ T cell SHIV provirus detection. For each animal, SHIV detection methods with detectable SHIV in any tested tissue or at any time point were indicated with a red "+", while those with undetectable values in all tested tissues and at all time points were indicated with a gray " - ". b Proportion of animals with detectable cell-free or cell-associated SHIV as defined by detectable SHIV plasma RNA, SHIV provirus in tissues or PBMCs, tissue mononuclear cell-associated infectious SHIV titers, or SHIV RNA-transcribing cells

and 2/6 animals becoming viremic, respectively (Fig. 3). Yet, this potential partial protection mediated by milk mAbs lacked statistical significance when compared to controls. Additionally, peak VLs, but not set point VLs, in DH378-treated, viremic animals were significantly higher than that of control animals and this difference was not attributable to more protective MHC types in the control animals (Table 1). This apparent mAb effect on plasma VL could be due to selection of more fit variants with subtle differences in time to peak VL not appreciated by our sampling schedule and/or antibody-dependent enhancement of viral infection of target cells. Antibody-dependent enhancement of HIV infection, observed as increased T/F variants and/or higher infection rates, has been previously associated with the presence of poorly neutralizing, HIV-specific antibodies similar to mAb DH378. ${ }^{48}$ However, peak VLs in the tri-mAb-treated, viremic animals were not elevated over those in control animals, indicating that a cocktail of functionally diverse mAbs may negate the potential effects of a single $\mathrm{mAb}$ on viral replication. Additionally, tri-mAb-treated animal LK81 demonstrated the lowest peak and set point VLs observed in any viremic animal (Fig. 3). This enhanced viral control post-acquisition may be attributable to the animal MHC background with two protective MHC alleles, Mamu-A001 and Mamu-B047a ${ }^{34-41}$; however, other viremic animals sharing the Mamu-A001 allele, including LK72 with two protective alleles, did not demonstrate notably lower VLs (Table 1). Furthermore, these MHC alleles are not associated with decreased peak VLs. ${ }^{34,40}$

Tissue-associated virus was assessed in viremic animals at 8 weeks via multiple approaches to fully characterize the state of the tissue-associated VL within lymphoid and Gl tract tissues (Fig. 4). The size of the resting memory cell latent reservoir in these infant RMs was not directly assessed owing to limitations of cell numbers. While limited differences in measures of virusinfected cells were observed between most infant RMs regardless of treatment groups, tri-mAb-treated animal LK81 did not have detectable proviral loads or tissue mononuclear cell-associated infectious viral titers, and only low levels of active viral transcription in only two of the five tissues tested. This finding is consistent with the low plasma viremia observed in this animal and potentially attributable to the pre-existing tri-mAb anti-HIV functionalities. While our characterizations of tissue-associated virus were otherwise similar between treatment groups, tissue mononuclear cell-associated infectious viral titers indicated that the tonsillar tissue had heightened infectious SHIV levels compared to other lymphoid and Gl tissues. This result aligns with previous reports indicating the susceptibility of tonsillar tissue as a portal of HIV entry and amplification ${ }^{49}$ and further suggests that the tonsils may be a key anatomical site for strategies seeking to prevent or treat HIV infections, particularly through the oral route.

Interestingly, we observed high proviral loads in a range of tissues from several control and DH378 mAb-treated nonviremic animals (Fig. 5). These findings indicate either abortive or very-low-level SHIV infection. Generation of pseudoviruses containing two proviral envelope genes (env) isolated from these animals demonstrated that they were capable of infection in vitro, indicating functional envelope genes of these proviruses. However, the viability of the full-length provirus was not assessed owing to inability to amplify the full genome. Notably, detectable provirus was not observed in CD4+ T cells isolated from any tissues of all non-viremic, tri-mAb-treated animals. This finding indicates that the tri-mAb cocktail successfully prevented or eliminated even abortive infection in these animals. Alternatively, Liu et al. recently demonstrated that passive bNAb infusion mediating complete protection from subsequent intravaginal SHIV challenge in RMs did not prevent initial virus acquisition and proliferation in distal tissues but rather that the bNAb successfully cleared this infection by around 10 days postchallenge. ${ }^{50}$ Similarly, Hessell et al. reported that $24-\mathrm{h}$ postexposure passive infusion of an anti-HIV bNab cocktail in SHIVchallenged infant RMs cleared virus from blood and tissues. ${ }^{26}$ Hessell et al. also demonstrated that passive infusion of RMs with a V2i-specific mAb prior to repeated SHIV challenges reduced blood VLs and lymphoid tissue proviral loads. ${ }^{51}$ Thus perhaps the various anti-SHIV functionalities mediated by the tri-mAb treatment in this study similarly cleared the initial infection of distal tissues. To determine whether the tri-mAb treatment prevented initial SHIV acquisition in these animals or mediated clearance of SHIV in distal tissues, additional work characterizing the viral populations present in distal lymphoid tissues at various early time points post-challenge is necessary. Regardless of the mechanism leading to uninfected distal 
tissues, we speculate that the potent ADCC-mediating mAb, DH382, may have played a pivotal role since the weakly neutralizing $\mathrm{DH} 378 \mathrm{mAb}$ treatment alone failed to similarly limit tissue-associated proviral loads.

Finally, the SHIV diversity at peak viremia in infant RMs was measured to assess the capability of these milk mAbs to limit the number of $T / F$ variants transmitted across the mucosa and/or early SHIV diversification after oral challenge (Table 2). The fewer distinct SHIV variants isolated from tri-mAb-treated animals compared to control mAb-treated animals indicates that this cocktail of maternal mAbs successfully limited the number of SHIV variants capable of sustaining infection in infant RMs. As DH378 mAb-treated infant RMs exhibited similar numbers of SHIV plasma variants compared to control mAb-treated animals, again the combination of distinct antibody functions, with a particular emphasis on ADCC, is likely important for reducing SHIV variant transmission. Of note, as only day 14 post-challenge SHIV variants were isolated to assess viral diversity, the mechanism of mAbmediated control of SHIV diversity may include limiting the number of initial T/F variants, minimizing postinfection diversification, or a combination of both.

In conclusion, a combination of mAbs with diverse Env epitope specificity, weak neutralization capabilities, and ADCC functionality appeared effective at lowering the number of T/F SHIV variants and potentially SHIV levels in circulating CD4+ T cells and tissues in SHIV-challenged infant RMs. Taken together, these trends suggest that polyclonal non-broadly neutralizing maternal mAbs with multiple functionalities, and particularly ADCC, may contribute to the naturally limited MTCT during breastfeeding, potentially through inhibition of initial virus acquisition at mucosal sites or through rapid viral clearance in the tissues. A better understanding of the mechanism of this protection as well as other maternal and/or infant factors that contribute to the relative protection of infants in the setting of breastfeeding may lead to more effective pediatric HIV therapeutic and prophylactic vaccine design strategies.

\section{MATERIALS AND METHODS}

More detailed description of experimental methods is provided in Supplemental Methods.

\section{Study design}

Twenty infant RMs (1-2 weeks old) were intravenously infused with either $10 \mathrm{mg} / \mathrm{kg}$ anti-HIV Env gp120 mAb DH378 $(n=6)$, $10 \mathrm{mg} / \mathrm{kg}$ anti-influenza HA mAb CH65 $(n=6), 30 \mathrm{mg} / \mathrm{kg}$ a-HA $\mathrm{mAb} \mathrm{CH} 65(n=2)$, or $30 \mathrm{mg} / \mathrm{kg}$ of a tri-mAb cocktail composed of stoichiometric equivalents of 3 anti-HIV Env gp120 mAbs $(n=6)-$ $\mathrm{DH} 377, \mathrm{DH} 378$, and $\mathrm{DH} 382-1 \mathrm{~h}$ prior to the first oral SHIV challenge. Animals were subjected to 3 oral challenges per day for 3 consecutive days consisting of 5000 TCID $_{50}$ SHIV-1157ipd3N4 (NIH AIDS Reagent Program) incubated in $1 \mathrm{~mL}$ of RPMI containing 1-3 $\mu \mathrm{g} / \mathrm{mL}$ DH378, CH65, or tri-mAb cocktail for $15 \mathrm{~min}$, followed by dilution in $\sim 10 \mathrm{~mL}$ formula feed to simulate oral acquisition via breastfeeding. Animals were re-infused with their respective antibody infusions 1 week after the initial infusion. Blood and saliva (via weck cell sponges) were collected before each infusion, $1 \mathrm{~h}$ after each infusion, 1 day after each infusion, and at weeks 2, 4, 6 , and 8 of the study. All animals were necropsied at week 8 of the study and tissues were collected.

Production of infusion mAbs

Infusion mAbs were obtained through antigen-specific B cell sorting and Ig variable gene amplification, as previously described, ${ }^{20}$ and produced through transient transfection either by the manufacturer Catalent (DH378; Catalent) or at the Duke Human Vaccine Institute in Expi293 cells (DH377, DH382, CH65), as previously described. ${ }^{52}$
HIV-1 neutralization in TZM-bl cells

NAb titers were measured by the reduction in Tat-regulated Luc reporter gene expression in a TZM-bl (NIH AIDS Reagent Program) reporter cell assay, as previously described. ${ }^{53}$

Tissue mononuclear cell viral coculture

Tissue-associated infectious virus titer was assessed through Tatregulated Luc-F reporter gene expression to quantify infection of TZM-bl reporter cells after coculture with serial dilutions of tissue mononuclear cells isolated from RMs.

Plasma viral RNA load quantification

qRT-PCR was performed to determine the SHIV-1157ipd3N4 RM plasma RNA load, as previously described. ${ }^{54}$

Mononuclear cell provirus quantification

RM CD4+ T cell-associated gDNA was isolated from various $\mathrm{Gl}$ and lymphoid tissues with the QIAaMP DNA/RNA Extraction Kit (Qiagen) and quantified using the Biorad QX200 droplet digital PCR System according to the manufacturer's instructions (Biorad) with SHIV gag-specific primers and probe and a commercially available human TERT-specific reference assay (Biorad). The SHIV proviral load in SHIV copies/million CD4+ T cells was calculated by dividing the SHIV DNA copy number by the TERT copy number divided by 2 multiplied by $10^{6}$ cells.

Measurement of virus-specific lgG levels in plasma and saliva Antibody concentrations in plasma and saliva were identified with ELISA, as previously described. ${ }^{28}$

Antibody-dependent cellular cytotoxicity ADCC activity of the purified mAbs and peripheral serum samples was determined by a luciferase-based cell killing assay with Renilla luciferase reporter gene encoding SHIV1157-ipd3N4-IMC, CEM. $\mathrm{NKR}_{\mathrm{CCR} 5}$ target cells (NIH AIDS Reagent Program) and PBMCs from a healthy HIV-seronegative donor as previously described. ${ }^{29,32}$

\section{Flow cytometry}

RM PBMCs or tissue mononuclear cells were stained with a panel of fluorochrome-conjugated antibodies and either phenotyped using an LSRII flow cytometer or bulk sorted into CD4+ and CD8+ $T$ cells using a FACS Aria II cytometer (BD Biosciences). CD4+ and $\mathrm{CD} 8+\mathrm{T}$ cells were positively selected from isolated tissue mononuclear cells by sequential selection of lymphocytes, FSC and SSC singlets, viable cells, CD45 + leukocytes, CD3 + T cells, and CD4+ vs CD8+ T cells. Data analysis was performed using the FlowJo software (TreeStar).

In situ hybridization

In situ hybridization for the detection and quantification of SHIV gag RNA in formalin-fixed tissue blocks was performed using the Affymetrix protocol according to the manufacturer's instruction (Affymetrix). Tissue blocks were stained for CD3, DAPI, and SHIV gag RNA and imaged on slides with a Leica TCS SP8 confocal microscope. The number of SHIV RNA-producing cells was reported as the number of SHIV gag RNA+ cells per 1000 CD3+ T cells.

\section{$\mathrm{T} / \mathrm{F}$ analysis}

T/F viral sequences in plasma were obtained by SGA through nested RT-PCR with subsequent direct amplicon sequencing, as previously described. ${ }^{55}$ Sequence alignments and phylogenetic trees were constructed using clustalW and Highlighter plots were created using the tool at www.lanl.gov.

To identify and enumerate T/F variants, clusters of related sequences were visually analyzed using phylogenetic trees (Figtree v1.4) and sequences containing $<2$ mutations were considered a single variant. Variants containing $\geq 2$ mutations 
were considered as progeny of distinct T/F genomes. Potential GA hymermutations caused by APOBEC $3 \mathrm{G} / 3 \mathrm{~F}$ were identified using Hypermut algorithm 2.0 and were reverted for analysis if there were $\leq 2$ present. Sequences that had $>3$ potential APOBEC $3 \mathrm{G} / 3 \mathrm{~F}$ mutations were not considered for $\mathrm{T} / \mathrm{F}$ analysis (Hypermut, http:// www.hiv.lanl.gov). Sequence clusters of $\geq 2$ sequences with $\geq 2$ shared mutations were considered as distinct $\mathrm{T} / \mathrm{F}$ variants.

Provirus env cloning and pseudovirus preparation

SHIV env amplification from gDNA extracted from infant RM CD4+ $\mathrm{T}$ cells was done through bulk-nested PCR and cloning into pcDNA3.1/N5-His-Topo (Invitrogen). Pseudovirus was produced via cotransfection of the SHIV env plasmid and a plasmid containing a subtype B env-deficient HIV genome (SG3Denv) in 293T cells (Invitrogen) as previously described. ${ }^{53}$ Pseudotyped virus infectivity was screened by TZM-bl cell infection and Tat-regulated luminescence with the Bright-Glo luciferase reagent (Promega).

\section{Statistical analysis}

Statistical tests were performed with SAS v9.4 (SAS Institute). Comparisons of viral load, proviral load (copies/million cells), and the number of $T / F$ variants in infants from each $\mathrm{mAb}$ treatment group were performed using the exact Wilcoxon test. The proviral load (binary designation) and the number of infants with detectable SHIV in each mAb treatment group were compared with Fisher's exact test. FDR $p$ value correction was used to correct for multiple comparisons. A $p$ value of $<0.05$ (two-tailed) was considered as significant for all analyses.

\section{Disclaimer}

The funders had no role in the study design, data collection and analysis, decision to publish, or preparation of the manuscript.

\section{ACKNOWLEDGEMENTS}

We thank Ruth Ruprect and the Dana Farber Cancer Institute for generously permitting the use of the SHIV-1157ipd3N4 for challenging the animals in this study through the NIH AIDS reagent Program. We also thank the Duke Human Vaccine Institute Protein Production Facility for help with mAb production, the Duke University Sequencing and Genomic Technology core facility for help with the ddPCR and Fluidigm assays, Barton Haynes and Kevin Saunders for providing protein antigens used in ELISAs, Tori Huffman and R. Whitney Edwards for technical assistance on ADCC data collection, David O'Connor and Roger Wiseman at the Wisconson National Primate Center for performing the MHC-typing, and the NIH HIV Research and Design (HIVRAD) and Research Project Grant (R01) programs for funding. TZM-bl cells and SG3 $\Delta$ env were provided by John Kappes and Xiaoyun Wu through the NIH AIDS Reagent Program. This work was funded by HHS/National Institutes of Health (R01Al1063980, P01Al117915).

\section{AUTHOR CONTRIBUTIONS}

S.P., J.E.H., J.P., R.G., and R.J.M. contributed to study design. J.E.H., R.G., R.J.M., A.K. T.L.J., Q.N.N., J.W.P., G.F., C.L., D.C.M., C.M., X.A.-H., F.S., and P.P.A. contributed to immunologic and virologic assays and data collection. J.E.H., R.G., R.J.M., J.A.E., H.H., F.S., and P.P.A. contributed to tissue processing and sample collection. J.W.P. produced study antibodies. J.E.H., R.G., R.J.M., S.P., F.S., and P.P.A. contributed to animal management. J.E.H., R.G., R.J.M., A.K., J.W.P., and S.R.P. drafted the manuscript. M.C. and N.A.V. performed power calculations and statistical analyses.

\section{ADDITIONAL INFORMATION}

The online version of this article (https://doi.org/10.1038/s41385-018-0067-7) contains supplementary material, which is available to authorized users.

Competing interests: The authors declare no competing interests.

\section{REFERENCES}

1. UNAIDS. Children and HIV: fact sheet, July 2016. Available from http://www. unaids.org/sites/default/files/media_asset/FactSheet_Children_en.pdf (2016).
2. Kourtis, A. P., Butera, S., Ibegbu, C., Belec, L. \& Duerr, A. Breast milk and HIV-1: vector of transmission or vehicle of protection? Lancet Infect. Dis. 3, 786-793 (2003).

3. Kuhn, L. \& Aldrovandi, G. Survival and health benefits of breastfeeding versus artificial feeding in infants of HIV-infected women: developing versus developed world. Clin. Perinatol. 37, 843-862 (2010).

4. American Academy of Pediatrics Work Group on Breastfeeding. Breastfeeding and the use of human milk. Pediatrics 100, 1035-1039 (1997). Available from https://doi.org/10.1542/peds.100.6.1035.

5. WHO. Mother-to-child transmission of HIV. Available from http://www.who.int/ hiv/topics/mtct/about/en/ (2017).

6. Nachega, J. B. et al. Adherence to antiretroviral therapy during and after pregnancy in low-income, middle-income, and high-income countries: a systematic review and meta-analysis. AIDS 26, 2039-2052 (2012).

7. Psaros, C., Remmert, J. E., Bangsberg, D. R., Safren, S. A. \& Smit, J. A. Adherence to HIV care after pregnancy among women in sub-Saharan Africa: falling off the cliff of the treatment cascade. Curr. HIV/AIDS Rep. 12, 1-5 (2015).

8. Drake, A. L., Wagner, A., Richardson, B. \& John-Stewart, G. Incident HIV during pregnancy and postpartum and risk of mother-to-child HIV transmission: a systematic review and meta-analysis. PLoS Med. 11, e1001608 (2014).

9. Moodley, D. et al. Incident HIV infection in pregnant and lactating women and its effect on mother-to-child transmission in South Africa. J. Infect. Dis. 203, 1231-1234 (2011).

10. Moodley, D., Esterhuizen, T. M., Pather, T., Chetty, V. \& Ngaleka, L. High HIV incidence during pregnancy: compelling reason for repeat HIV testing. AIDS 23, 1255-1259 (2009).

11. Fouda, G. G. \& Permar, S. R. Immune-based interventions to prevent postnatal HIV-1 transmission. Trends Microbiol. 22, 425-427 (2014).

12. Saeland, E. et al. MUC1 in human milk blocks transmission of human immunodeficiency virus from dendritic cells to T cells. Mol. Immunol. 46, 2309-2316 (2009).

13. Kuhn, L. et al. Alpha-defensins in the prevention of HIV transmission among breastfed infants. J. Acquir. Immune Defic. Syndr. 39, 138-142 (2005).

14. Harmsen, M. C., Swart, P. J., de Bethune, M. P., Pauwels, R. \& De Clercq, E. The TH et al. Antiviral effects of plasma and milk proteins: lactoferrin shows potent activity against both human immunodeficiency virus and human cytomegalovirus replication in vitro. J. Infect. Dis. 172, 380-388 (1995).

15. Villamor, E. et al. Long-chain n-6 polyunsaturated fatty acids in breast milk decrease the risk of HIV transmission through breastfeeding. Am. J. Clin. Nutr. 86, 682-689 (2007).

16. Walter, J. et al. High concentrations of interleukin 15 in breast milk are associated with protection against postnatal HIV transmission. J. Infect. Dis. 200, 1498-1502 (2009).

17. Mansour, R. G. et al. The presence and anti-HIV-1 function of tenascin C in breast milk and genital fluids. PLOS ONE 11, e0155261 (2016).

18. Martin, V., Maldonado, A., Fernandez, L., Rodriguez, J. M. \& Connor, R. I. Inhibition of human immunodeficiency virus type 1 by lactic acid bacteria from human breastmilk. Breastfeed. Med. 5, 153-158 (2010).

19. Fouda, G. G. et al. HIV-specific functional antibody responses in breast milk mirror those in plasma and are primarily mediated by IgG antibodies. J. Virol. 85, 9555-9567 (2011).

20. Sacha, C. R. et al. Restricted isotype, distinct variable gene usage, and high rate of gp120 specificity of HIV-1 envelope-specific B cells in colostrum compared with those in blood of HIV-1-infected, lactating African women. Mucosal Immunol. 8 316-326 (2015).

21. Tuaillon, E. et al. Human milk-derived B cells: a highly activated switched memory cell population primed to secrete antibodies. J. Immunol. 182, 7155-7162 (2009).

22. Mabuka, J., Nduati, R., Odem-Davis, K., Peterson, D. \& Overbaugh, J. HIV-specific antibodies capable of ADCC are common in breastmilk and are associated with reduced risk of transmission in women with high viral loads. PLoS Pathog. 8, e1002739 (2012).

23. Milligan, C., Richardson, B. A., John-Stewart, G., Nduati, R. \& Overbaugh, J. Passively acquired antibody-dependent cellular cytotoxicity (ADCC) activity in HIVinfected infants is associated with reduced mortality. Cell Host Microbe 17, 500-506 (2015).

24. Mascola, J. R. \& Haynes, B. F. HIV-1 neutralizing antibodies: understanding nature's pathways. Immunol. Rev. 254, 225-244 (2013).

25. Baba, T. W. et al. Human neutralizing monoclonal antibodies of the $\lg G 1$ subtype protect against mucosal simian-human immunodeficiency virus infection. Nat. Med. 6, 200-206 (2000).

26. Hessell, A. J. et al. Early short-term treatment with neutralizing human monoclonal antibodies halts SHIV infection in infant macaques. Nat. Med. 22, 362-368 (2016). 
Polyclonal HIV envelope-specific breast milk antibodies limit founder... JE Himes et al.

1726

27. Gray, E. S. et al. The neutralization breadth of HIV-1 develops incrementally over four years and is associated with CD4+ T cell decline and high viral load during acute infection. J. Virol. 85, 4828-4840 (2011).

28. Jeffries, T. L. et al. The function and affinity maturation of HIV-1 gp120-specific monoclonal antibodies derived from colostral B cells. Mucosal Immunol. 9, 414-427 (2016).

29. Pollara, J. et al. Association of HIV-1 envelope-specific breast milk IgA responses with reduced risk of postnatal mother-to-child transmission of HIV-1. J. Virol. 89, 9952-9961 (2015).

30. Song, R. J. et al. Molecularly cloned SHIV-1157ipd3N4: a highly replicationcompetent, mucosally transmissible R5 simian-human immunodeficiency virus encoding HIV clade C Env. J. Virol. 80, 8729-8738 (2006).

31. Martinez, D. R. et al. Maternal binding and neutralizing lgG responses targeting the $C$ terminal region of the V3 loop are predictive of reduced peripartum HIV-1 transmission risk. J. Virol. https://doi.org/10.1128/JVI02422-16 (2017).

32. Permar, S. R. et al. Maternal HIV-1 envelope-specific antibody responses and reduced risk of perinatal transmission. J. Clin. Invest. 125, 2702-2706 (2015).

33. Siddappa, N. B. et al. R5 clade C SHIV strains with tier 1 or 2 neutralization sensitivity: tools to dissect env evolution and to develop AIDS vaccines in primate models. PLOS ONE 5, e11689 (2010).

34. Loffredo, J. T. et al. Mamu-B*08-positive macaques control simian immunodeficiency virus replication. J. Virol. 81, 8827-8832 (2007).

35. Mothe, B. R. et al. Expression of the major histocompatibility complex class I molecule Mamu- $A^{*} 01$ is associated with control of simian immunodeficiency virus SIVmac239 replication. J. Virol. 77, 2736-2740 (2003).

36. Muhl, T., Krawczak, M., Ten Haaft, P., Hunsmann, G. \& Sauermann, U. MHC class I alleles influence set-point viral load and survival time in simian immunodeficiency virus-infected rhesus monkeys. J. Immunol. 169, 3438-3446 (2002).

37. O'Connor, D. H. et al. Major histocompatibility complex class I alleles associated with slow simian immunodeficiency virus disease progression bind epitopes recognized by dominant acute-phase cytotoxic-T-lymphocyte responses. J. Virol. 77, 9029-9040 (2003).

38. Pal, R. et al. ALVAC-SIV-gag-pol-env-based vaccination and macaque major histocompatibility complex class I $\left(A^{*} 01\right)$ delay simian immunodeficiency virus SIVmac-induced immunodeficiency. J. Virol. 76, 292-302 (2002).

39. Sauermann, U. et al. MHC class I haplotypes associated with survival time in simian immunodeficiency virus (SIV)-infected rhesus macaques. Genes Immun. 9, 69-80 (2008).

40. Yant, L. J. et al. The high-frequency major histocompatibility complex class I allele Mamu-B*17 is associated with control of simian immunodeficiency virus SIVmac239 replication. J. Virol. 80, 5074-5077 (2006).
41. Zhang, Z. Q. et al. Mamu- $A^{*} 01$ allele-mediated attenuation of disease progression in simian-human immunodeficiency virus infection. J. Virol. 76, 12845-12854 (2002).

42. Albrecht, C. et al. Progression to AIDS in SIV-infected rhesus macaques is associated with distinct KIR and MHC class I polymorphisms and NK cell dysfunction. Front. Immunol. 5, 600 (2014).

43. Santra, S. et al. Human non-neutralizing HIV-1 envelope monoclonal antibodies limit the number of founder viruses during SHIV mucosal infection in rhesus macaques. PLoS Pathog. 11, e1005042 (2015).

44. Becquet, R. et al. Children who acquire HIV infection perinatally are at higher risk of early death than those acquiring infection through breastmilk: a meta-analysis. PLOS ONE 7, e28510 (2012).

45. $\mathrm{Ng}, \mathrm{C}$. T. et al. Passive neutralizing antibody controls SHIV viremia and enhances $B$ cell responses in infant macaques. Nat. Med. 16, 1117-1119 (2010).

46. Ghulam-Smith, M. et al. Maternal but not infant anti-HIV-1 neutralizing antibody response associates with enhanced transmission and infant morbidity. mBio $\mathbf{8}$, e01373-17 (2017).

47. Polonis, V. R. et al. Recent advances in the characterization of HIV-1 neutralization assays for standardized evaluation of the antibody response to infection and vaccination. Virology 375, 315-320 (2008).

48. Gorlani, A. \& Forthal, D. N. Antibody-dependent enhancement and the risk of HIV infection. Curr. Hiv. Res. 11, 421-426 (2013).

49. Stahl-Hennig, C. et al. Rapid infection of oral mucosal-associated lymphoid tissue with simian immunodeficiency virus. Science 285, 1261-1265 (1999).

50. Liu, J. et al. Antibody-mediated protection against SHIV challenge includes systemic clearance of distal virus. Science 353, 1045-1049 (2016).

51. Hessell, A. J. et al. Reduced cell-associated DNA and improved viral control in macaques following passive transfer of a single anti-V2 monoclonal antibody and repeated SHIV challenges. J. Virol. https://doi.org/10.1128/JVI.02198-17 (2018).

52. Bonsignori, M. et al. Staged induction of HIV-1 glycan-dependent broadly neutralizing antibodies. Sci. Transl. Med. https://doi.org/10.1126/scitranslmed.aai7514 (2017).

53. Li, M. et al. Human immunodeficiency virus type 1 env clones from acute and early subtype B infections for standardized assessments of vaccine-elicited neutralizing antibodies. J. Virol. 79, 10108-10125 (2005).

54. Wilks, A. B. et al. High cell-free virus load and robust autologous humoral immune responses in breast milk of simian immunodeficiency virus-infected African green monkeys. J. Virol. 85, 9517-9526 (2011).

55. Keele, B. F. et al. Low-dose rectal inoculation of rhesus macaques by SIVsmE660 or SIVmac251 recapitulates human mucosal infection by HIV-1. J. Exp. Med. 206, 1117-1134 (2009). 